\title{
MAN/MACHINE INTERFACE BASED ON THE DISCHARGE TIMINGS OF SPINAL MOTOR NEURONS AFTER TARGETED MUSCLE REINNERVATION
}

Dario Farina ${ }^{1,2^{*}}$, Ivan Vujaklija ${ }^{1,2}$, Massimo Sartori ${ }^{2+}$, Tamás Kapelner $^{2+}$, Francesco Negro $^{2,3}$, Ning Jiang $^{4}$, Konstantin Bergmeister ${ }^{5,6}$, Arash Andalib ${ }^{7}$, Jose Principe ${ }^{7}$, and Oskar C. Aszmann ${ }^{5,6}$

${ }^{1}$ Department of Bioengineering, Imperial College London, London, UK

${ }^{2}$ Clinic for Trauma Surgery, Orthopaedic Surgery and Plastic Surgery - Research Department of Neurorehabilitation Systems, University Medical Center Göttingen, Göttingen, Germany

${ }^{3}$ Department of Clinical and Experimental Sciences, University of Brescia, 25123 Brescia, Italy

${ }^{4}$ Department of Systems Design Engineering, Faculty of Engineering, University of Waterloo, Waterloo, CANADA

${ }^{5}$ Christian Doppler Laboratory for Restoration of Extremity Function, Medical University of Vienna, AUSTRIA

${ }^{6}$ Division of Plastic and Reconstructive Surgery, Department of Surgery, Medical University of Vienna, AUSTRIA

${ }^{7}$ Department of Electrical and Computer Engineering, University of Florida, Gainesville, FL, USA

†These authors contributed equaly to this work 


\section{Abstract}

The intuitive control of upper-limb prostheses requires a man/machine interface that directly exploits biological signals. Here, we define and experimentally test an offline man/machine interface that takes advantage of the discharge timings of spinal motor neurons. The motor-neuron behaviour is identified by deconvolution of the electrical activity of muscles reinnervated by nerves of a missing limb in patients with amputation at the shoulder or humeral level. We mapped the series of motorneuron discharges into control commands across multiple degrees of freedom via the offline application of direct proportional control, pattern recognition and musculoskeletal modelling. A series of experiments performed on six patients reveal that the man/machine interface has superior offline performance than conventional direct electromyographic control applied after targeted muscle innervation. The combination of surgical procedures, decoding and mapping into effective commands constitutes an interface with the output layers of the spinal cord circuitry that allows for the intuitive control of multiple degrees of freedom. 
Natural and intuitive control of upper limb prostheses requires the establishment of a man-machine interface that explores the perception-action cycle directly based on biological signals $^{1-3}$. These signals are processed to extract information about the user's intent and are translated into commands for the prosthesis. The neuromuscular system can be interfaced at various levels to extract neural signals that code the intended movement, e.g., via brain, nerve or muscle recordings ${ }^{4-9}$. Although direct brain signal decoding provides the neural information associated to movement intention and control ${ }^{10-12}$, peripheral approaches (nerves or muscles) are so far the only clinically viable solutions for re-establishing upper limb function in amputees ${ }^{4,13}$. In these patients, the availability of nerve and muscle structures above the amputation allows access to neural information at the output of the spinal cord circuitries.

Muscle interfacing is generally possible only when relevant remnant muscle tissue is available following the amputation. The higher the amputation level, the greater the need for control signals, with fewer muscles available to interface for intuitive control. Nonetheless, muscles can be denervated and reinnervated by nerves that used to carry the neural code to the missing limb ${ }^{14}$. This procedure is known as targeted muscle reinnervation $(T M R)^{2,15,16}$ and it constituted a breakthrough in prosthetics ${ }^{17} . \ln T M R$, muscles serve as biological amplifiers of nerve activity because of the association between the action potentials discharged by the efferent nerve fibers (axons of motor neurons) and the action potentials of the innervated muscle fibers. Each nerve action potential is transduced into a compound muscle fiber action potential that carries the same neural information, coded by its timing of occurrence. 
TMR and electromyographic (EMG) recordings from reinnervated muscles determine a man-machine interface that, in principle, allows the indirect detection (from muscle signals) of the ensemble efferent activity of any nerve once it is directed to an accessible target muscle. However, the classic use of TMR and EMG for man-machine interfacing does not aim at decoding the underlying neural information (timings of occurrence of action potentials) sent to muscles by motor neurons. Rather, this interface uses the EMG as an interferent signal (colored noise) from which few global features are extracted ${ }^{18}$, e.g. amplitude or spectral moments, for either direct control or for identifying sets of predefined movements ${ }^{19}$. This global approach is common to previous TMR investigations, even when more advanced (multi-channel) EMG systems have been employed ${ }^{19}$. This procedure limits the decoding quality of the neural interface because the global EMG is a spatio-temporal summation of action potentials that creates correlations amongst multiple channels and therefore determines an ill posed inverse problem for decoding ${ }^{20}$. Similarly, control methods based on more selective intramuscular EMG recordings have been so far based on global EMG analysis without decoding the contributions of individual motor neurons ${ }^{21,22}$ or on a very small number of decoded motor neurons (e.g., two experimentally decoded motor neurons from intramuscular EMG signals $\mathrm{in}^{23}$ ). Decoupling the neural information contained in the EMG signals, which exactly correspond to the timings of discharge of the efferent nerve fibers, from the shapes of the muscle fiber action potentials would determine a direct interface with the spinal motor neurons.

Here we describe a neural interface that, following $\mathrm{TMR}^{24,25}$, extracts the sources of neural information - the discharge timings of motor neurons - through EMG deconvolution. The decoded neural information is then mapped into effective commands for intuitive prosthetic control. The effectiveness of the decoded neural information potential prosthesis 
control is demonstrated offline by associating the activity of motor neurons innervating the missing limb of amputees with the kinematics of intended motor tasks. We experimentally validate this association for multiple degrees of freedom in six patients following TMR. Therefore, here we present and substantiate the concept of a man-machine interface that extracts neural information sent from the output spinal cord circuitries by a combination of surgical procedures, advanced neural decoding, and mapping into multiple degrees of freedom.

\section{Results}

The approach is based on recording multi-channel ( $>50$ channels) EMG signals from reinnervation sites following TMR. The EMG signals are decoded by using a blind source separation method that separates the timings of activation of each motor unit from the waveforms representing the muscle fiber action potentials. The decoding thus provides multiple series of discharge timings of motor neurons reinnervating the target muscles. The discharge timings are projected into degrees of freedom by various mapping methods. Fig. 1 schematically presents the processing steps common to all the analyses performed. Three main experimental tests were conducted on TMR patients (Table 1) to present and validate the decoding and mapping approach. The aim of each experiment was to highlight different characteristics of the proposed approach. Experiment 1 presents the classification of the motor neuron activity into discrete classes. Experment 2 shows proportional control based on motor neuron discharges. Finally, Experiment 3 presents simulataneous and proportional mapping over multiple degrees of freedom.

\section{Experiment 1}


Experiment 1 was performed on patients T1, T2, and T3 (Table 1) and was designed to compare the use of motor neuron discharge timings with respect to classic global EMG features for the classification of intended tasks into a pre-defined set of classes (motor tasks). For this purpose, the EMG signals recorded from the reinnervation sites ( 6 reinnervation sites for patient $\mathrm{T} 1,4$ for $\mathrm{T} 2$, and 5 for T3; Table 1) were decomposed (decoded) into the contributions of individual motor units, separating the discharges of the innervating motor neurons from the waveforms of the muscle fiber action potentials. The discharge timings were then pooled to obtain series of ensemble discharge timings for the muscle regions where the recording EMG electrode grids were located (the area covered by each recording EMG grid was divided into four muscle regions). These series of ensemble discharges represented the neural drive sent to the corresponding muscle regions and were used as features for offline classification into 9, 7, and 11 classes (movements) for the three patients investigated.

The average number of motor units identified by decomposition of the multi-channel EMG signals in each reinnervation site during the executed tasks was $23.1 \pm 11.2$ (mean $\pm S D$, average over the three patients and all reinnervation sites and tasks). 
Table I. Characteristics of the patients investigated

\begin{tabular}{|c|c|c|c|c|c|c|}
\hline Patient & $\mathrm{T} 1$ & $\mathrm{~T} 2$ & T3 & T4 & T5 & T6 \\
\hline Age & 25 & 32 & 40 & 31 & 17 & 51 \\
\hline Sex & $M$ & M & $M$ & $M$ & $M$ & $M$ \\
\hline Amputation & $\begin{array}{c}\text { Gleno- } \\
\text { humeral right }\end{array}$ & $\begin{array}{c}\text { Gleno- } \\
\text { humeral left }\end{array}$ & $\begin{array}{c}\text { Gleno- } \\
\text { humeral left }\end{array}$ & $\begin{array}{c}\text { Trans- } \\
\text { humeral left }\end{array}$ & $\begin{array}{c}\text { Trans- } \\
\text { humeral left }\end{array}$ & $\begin{array}{c}\text { Trans- } \\
\text { humeral left }\end{array}$ \\
\hline $\begin{array}{l}\text { Time since } \\
\text { amputation }\end{array}$ & $\begin{array}{l}3 \text { years, } \\
2 \text { months }\end{array}$ & $\begin{array}{l}3 \text { years, } \\
2 \text { months }\end{array}$ & $>5$ years & $\begin{array}{l}2 \text { years, } \\
3 \text { months }\end{array}$ & $>5$ years & $>10$ years \\
\hline $\begin{array}{c}\text { Time since TMR } \\
\text { surgery }\end{array}$ & 10 months & 9 months & $\begin{array}{l}1 \text { year, } \\
5 \text { months }\end{array}$ & 9 months & $\begin{array}{l}4 \text { years, } \\
2 \text { months }\end{array}$ & $\begin{array}{l}4 \text { years, } \\
4 \text { months }\end{array}$ \\
\hline $\begin{array}{l}\text { Nerve in } m \text {. } \\
\text { pectoralis major } \\
\text { clavicular head }\end{array}$ & $\begin{array}{l}\text { musculo- } \\
\text { cutaneous }\end{array}$ & ulnaris & $\begin{array}{l}\text { musculo- } \\
\text { cutaneous }\end{array}$ & - & - & - \\
\hline $\begin{array}{c}\text { Nerve in } m \text {. } \\
\text { pectoralis major } \\
\text { sternocostal part }\end{array}$ & medianus & medianus & medianus & - & - & - \\
\hline $\begin{array}{c}\text { Nerve in } \\
m . \text { pectoralis } \\
\text { major abdominal } \\
\text { part }\end{array}$ & medianus & - & medianus & - & - & - \\
\hline $\begin{array}{l}\text { Nerve in } \\
\text { m. pectoralis } \\
\text { minor }\end{array}$ & ulnaris & medianus & ulnaris & - & - & - \\
\hline $\begin{array}{l}\text { Nerve in } \\
\text { m. latissimus } \\
\text { dorsi }\end{array}$ & radialis & radialis & radialis & - & - & - \\
\hline $\begin{array}{c}\text { Nerve in } \\
m . \text { infraspinatus }\end{array}$ & radialis & - & - & - & - & - \\
\hline $\begin{array}{c}\text { Nerve in } \\
m . \text { Biceps } \\
\text { brachii } \\
\text { caput longum }\end{array}$ & - & - & - & medianus & medianus & medianus \\
\hline $\begin{array}{c}\text { Nerve in } \\
\text { m. Biceps } \\
\text { brachii } \\
\text { caput breve }\end{array}$ & - & - & - & ulnaris & ulnaris & ulnaris \\
\hline $\begin{array}{l}\text { Nerve in } m . \\
\text { caput laterale } \\
\text { tricipitis }\end{array}$ & - & - & - & radialis & radialis & radialis \\
\hline
\end{tabular}




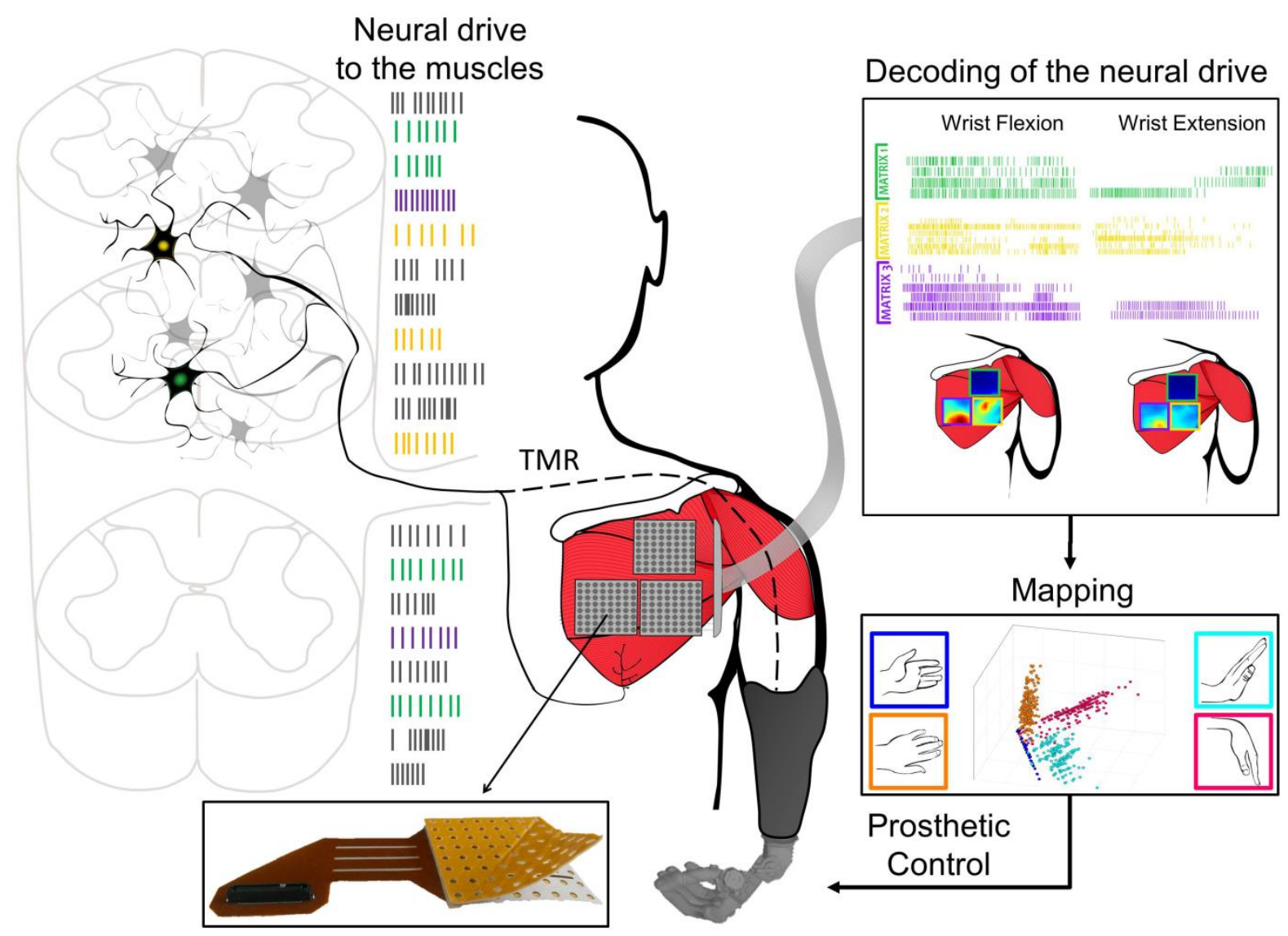

Figure 1 - Interfacing spinal motor neurons in humans. Nerves are surgically re-directed to innervate accessory muscles used as biological amplifiers of nerve activity by targeted muscle reinnervation (TMR). The discharge timings of the innervating motor neurons are decoded by deconvolution of the surface electromyographic signals. The series of discharge timings are then mapped into degrees of freedom. The mapping is obtained by diferent approaches, demonstrated in this study with three experiments. The interfacing provides access to the output from the spinal cord, as schematically represented by the colors of the series of discharges. The discharge patterns shown here are extracted from data acquired from patient T1. These discharge timings are represented here with the sole purpose of describing the general concept proposed in this work.

There were no significant differences in the number of decoded motor units nor in the accuracy of decoding between reinnervation sites, an observation that was valid for all experiments and all patients. This result is in agreement with several previous studies on EMG decoding in able-bodied individuals across a variety of muscles and contraction types ${ }^{26}$. The use of motor neuron discharge timings for motion classification provided an almost perfect classification accuracy of $>97 \%$ as an average over the three patients (Fig. 2). In the current experimental conditions, this performance was superior to both the use of the EMG 
root mean square (RMS) (average accuracy of $71 \%$ across all patients), and the use of RMS

together with time domain (TD) features (average accuracy of 85\%). The difference was

particularly evident for patient T3, who could execute the greatest number of tasks (11

classes) and for whom the classification accuracy was only 70\% when using the classic EMG

features and $>95 \%$ when using the decoded motor neuron discharge timings (Fig. 2). Fig. 2

presents the confusion matrices for the classification.



Figure 2 - Confusion matrices for the classification of motions of patients T1, T2 and T3 when using features extracted from global EMG analysis (root mean square (RMS), RMS and time domain (TD)) and when using the neural information as motor neuron discharge timings. The classified tasks are: elbow extension/flexion (EE/EF), hand close/open (HC/HO), wrist extension/flexion (WE/WF), wrist pronation/supination (WP/WS), thumb abduction/adduction (TAb/TAd), thumb extension/flexion (TE/TF), and no movement (NoM). The color scale represents the accuracy (\%) in discrimination between pairs of classes in the confusion matrices.

Fig.3 highlights one of the reasons for the effective discrimination of motor tasks based on motor neuron discharge timings with respect to global EMG features. The action potentials of three motor units are representatively shown as detected over the surface of 
the pectoralis muscle in subject T1 during hand closing and wrist supination. The spatial mapping of the global EMG amplitude and the action potentials of the motor units are presented. The global EMG amplitude, which is shown as an amplitude map (Fig. 3a), is similar for the two tasks, which are therefore difficult to discriminate using this feature. The spatial distribution of amplitude is indeed localized in the same area, represented by the upper left corner of the spatial amplitude maps in Fig. 3a. Therefore, the two tasks are executed by a neural drive reaching similar regions of the reinnervated muscles. A direct control of these two tasks in a physiological way (i.e., with natural muscle contractions corresponding to the two tasks) would not be possible because of this overlap. Classification of these two classes with global EMG features is, however, possible to some extent since classification uses features that may differ between the two tasks. Nonetheless, the similarity of the two EMG amplitude maps and the variability in execution over multiple trials reduces the classification performance when using global features. The spatialtemporal structure of the active motor units during the tasks is conversely very different and can be used for more robust discrimination. 


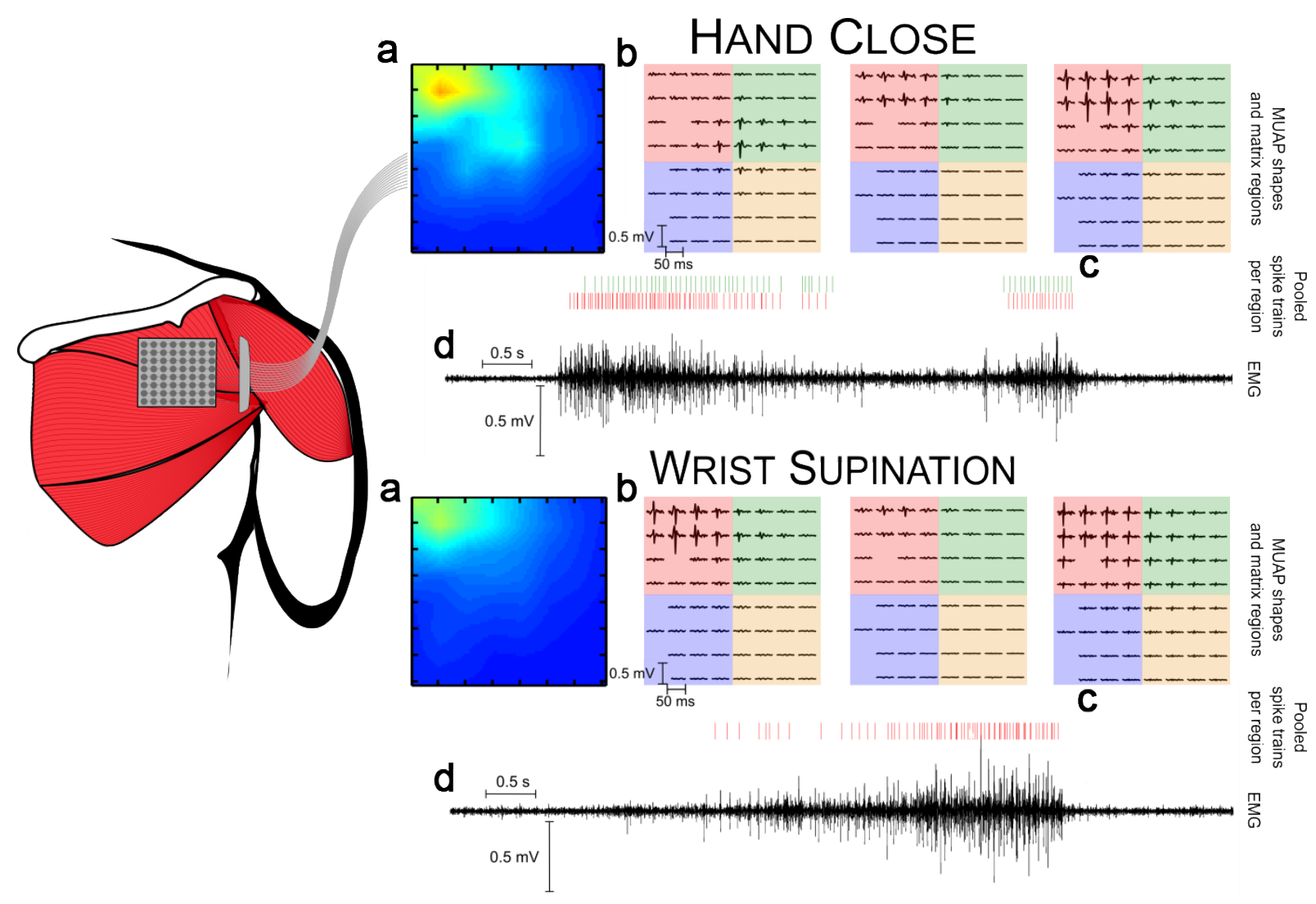

Figure 3 - Single channel electromyographic (EMG) recordings obtained from subject T1 during hand close and wrist supination of the phantom limb. (a) The colormap images represent the EMG root mean square (RMS) value for all electrodes of the matrix used for recording. (b) The EMG was decomposed into individual motor units. For clarity, only three representative motor units per task are represented with their multi-channel action potentials. The locations of the channels in the multichannel action potentials are coded with four colors. They represent an arbitrary partinioning applied to the grid to assign motor units to different regions of the reinnervated muscles. Each motor unit was assigned one of the four regions, according to the location of the peak value of the action potential waveforms. (c) The discharge timings of the motor units were pooled into ensemble discharge timings. These ensemble motor neuron discharges represent the neural drives reaching the four muscle regions. (d) Single-channel EMG signals synchronized with the discharge timings of motor neurons. In this example, the task "hand close" is performed with motor units assigned to the green and red portions of the grid, therefore the pooled discharge patterns are shown in green and red, respectively, for this task. Conversely, "wrist supination" is performed with motor units all detected in the red portion of the grid. The colormaps for surface EMG amplitude are relatively similar between the two tasks, whereas the motor unit activity is different and represented by neural drives reaching different regions of the reinnervated muscles. This representative data explain the reason for the superior classification performance obtained when using motor neuron discharge timings with respect to global EMG features. In all cases, poor discrimination by surface EMG was due to similar EMG amplitude spatial distribution between two or more classes whereas the motor neuron pooled discharge timings differed between classes, as in the example reported here. 
This experiment showed that the extraction of the timings of discharge of motor neurons may be beneficial for discriminating tasks of the missing limb with respect to global EMG feature classification. In the proposed approach, contrary to the global EMG that represents an average activity over the skin surface, each motor neuron may provide discriminating information from its series of discharges as well as from the location of the innervated muscle fibers.

\section{Experiment 2}

Experiment 2 (patients T4, T5, and T6) was designed to test the use of motor neuron discharge timings for proportional control. This has implications in methods for direct control following TMR. The tests were done on a single degree of freedom which was mapped proportionally from global EMG amplitude and from motor neuron discharge timings during slow-varying force contractions, in the full range of muscle activation. In this experiment, the average number of motor units identified by decomposition of the multichannel EMG signals at the observed reinnervation site across all three patients was $11.8 \pm$ 3.8. Fig. 4 presents an example of slow linear increase and decrease in intensity of activation by patient T4. Decomposition of the generated signals qualitatively revealed the two physiological mechanisms for increasing the level of activation: recruitment of additional motor neurons and modulation of the discharge frequency of the active motor neurons (Fig. 4c). It was also evident that the information in the population activity of motor neurons was highly correlated to the intensity of muscle activity and this was repeatable over different trials of the same task (Fig. 4e). 

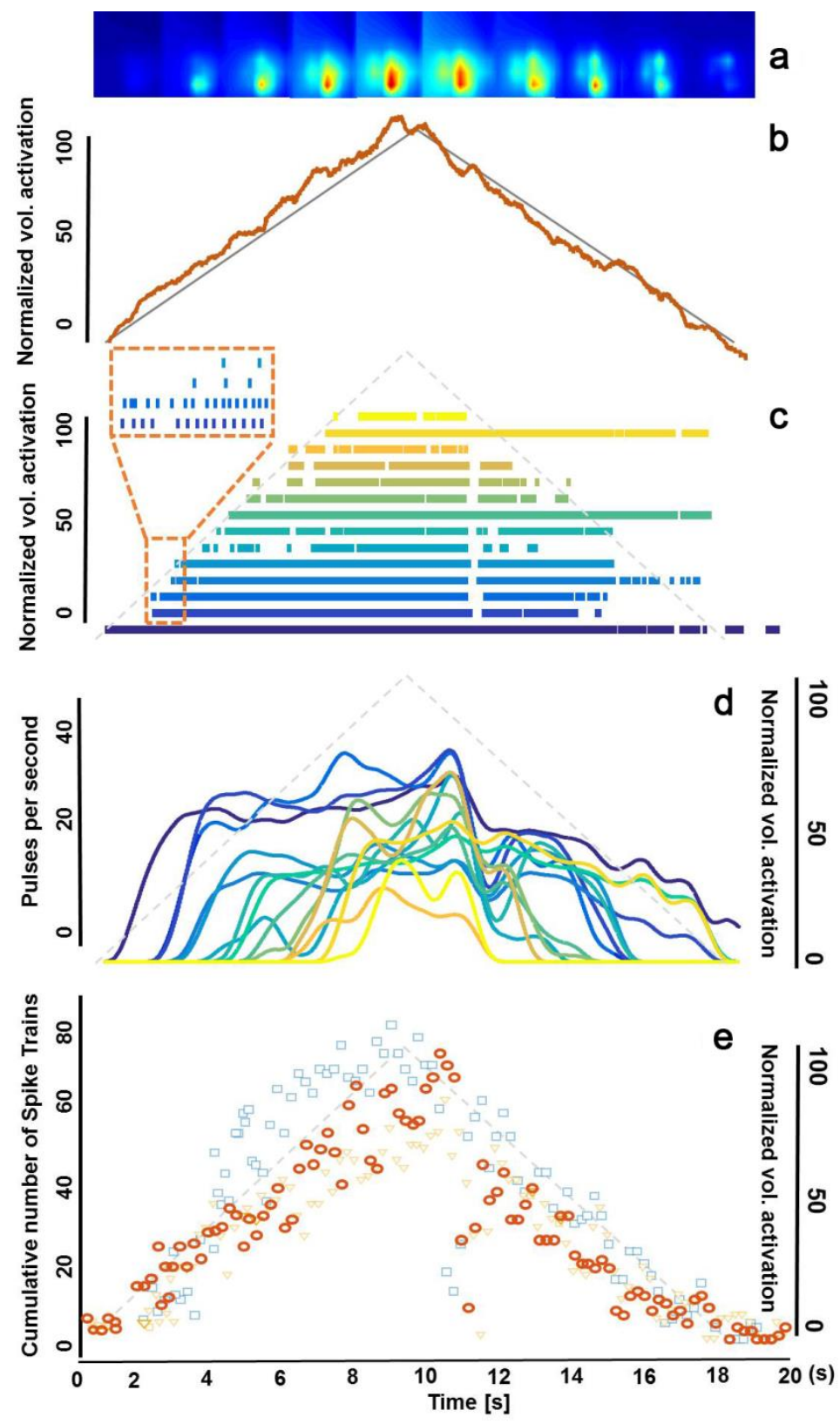

Figure 4 - Motor neuron behavior during linearly increasing and decreasing intensity of activation in patient T4. (a) Amplitude maps of the interference electromyographic (EMG) activity during a contraction of increasing and decreasing force (linear). (b) The cue that was given to the patient as visual feedback to modulate the intensity of the contraction is shown as a black line. The surface EMG amplitude is shown as a red line. (c) Discharge timings of motor neurons decoded through EMG decomposition. (d) Smoothed discharge rates of individual motor neurons with respect to the cue. (e) Instantaneous discharge rate estimated over intervals of 200 ms computed from the ensemble of motor neuron discharges for three repetitions of the same task (circles; each repetition is represented with a different color), showing the association between the instantaneous rate of motor neurons and intensity of activity. The behavior of motor neurons during the ramp contractions in this representative example fully reflects the general observation on all patients tested and all trials. This is evident from the results on each patient shown in Fig. 5. 
The representative results shown in Fig. 4 were confirmed in the three tested

patients. Fig. 5 shows representative estimates of contraction intensity for one patient as well as the individual results for each patient. The motor neuron discharge timings have been pooled to obtain the ensemble of discharge timings of all motor neurons, as an estimate of the neural drive. This estimate was compared with the global EMG amplitude. The estimates using motor neuron discharge timings outperformed those obtained with surface EMG for a large range of processing intervals and for all patients (Fig. 5).


Figure 5 - (a) Force estimates for patient T4, based on the envelope of the electromyographic (EMG) signals (blue) and motor neuron discharge timings (red) with respect to the cue (black). (b) Force estimates for patient T4 with post processing based on averaging over three consecutive past intervals. (c) Standard deviation $(\sigma)$ of estimation (color coded for each of the three patients T4-T6) after linear de-trending when varying the duration of the processing interval. (d) Standard deviation of estimation when applying the post processing to force estimates. 
This experiment showed that it is possible to extract direct proportional commands from motor neuron discharge timings and these commands are more accurate than those obtained with classic EMG amplitude.

\section{Experiment 3}

Finally, a direct mapping between decoded motor neuron discharge rates and kinematics was performed in Experiment 3, only for patient T6. The mapping into mechanical function was performed with two methods, either signal- or model-based. The signal-based approach applied a dimensionality reduction to the motor neuron discharge timing series without requiring kinematic labelling. The model-based approach projected the series of discharge timings into degrees of freedom by a forward biomechanical estimation of joint moments.

The signal-based approach was applied to 244 unsorted extended sources (over all reinnervaton sites) during a recording of $30 \mathrm{~s}$. The sources were extended by the EMG decomposition approach (see Methods) and all extensions were maintained for this estimation approach, contrary to all other methods presented for which the extended sources were not used. The extensions of the sources represent redundant information, but redundancy is exploited to define appropriate subspace projections with this approach. Due to the inclusion of extended sources for this approach, the number of sources used (244) was greater than for all other methods.

Fig. 6 shows the analysis made on three trials of the same composite movement with three concurrently active degrees of freedom. The third trial was used for test purposes, after calibration in the first two trials. The estimate on the train/test trials in this representative example corresponded to an average $R^{2}$ in the estimation of kinematics of 
$0.87 / 0.73$. It has to be noted that it is impossible to guarantee that the mirror movements were identical for the two sides and therefore the obtained $R^{2}$ is an underestimate of the actual performance. The performance obtained with three concurrently active degrees of freedom was similar to that obtained for two active degrees of freedom with an average $R^{2}$ of $0.65 / 0.72$, and for one degree of freedom $0.81 / 0.76$, for train and test trials, respectively. Fig. 6 shows the quality of the matching.

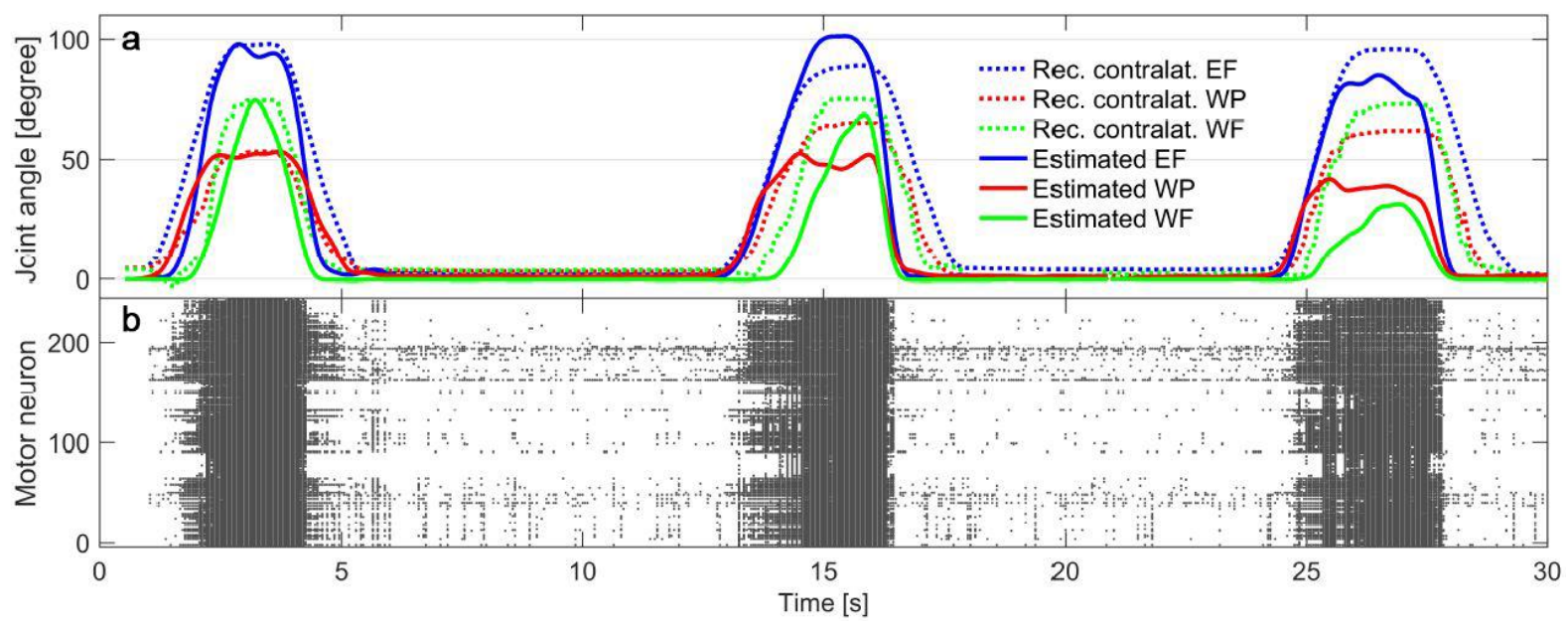

Figure 6 - (a) Performance of signal-based estimation on projecting three degrees of freedom composite movements. The dotted lines represent the recorded (Rec.) kinematics of the contraleral (contralat.) side for patient T6, while the solid ones depict the first three principal components after VARIMAX rotation (see Methods) which estimate elbow flexion (EF) in blue, wrist pronation (WP) in red, and wrist flexion (WF) in green. (b) The raster plot of the corresponding 244 motor neuron discharge timings (with the delayed replicas extracted by decomposition of electromyographic signals; see Methods). The first two tasks have been used for calibration and the last for testing.

The second mapping approach in this experiment was based on biomechanical modeling. Neural data-driven musculoskeletal modeling was used to demonstrate the possibility of reconstructing the neuro-mechanical function of the patient's phantom limb. This approach enabled translating motor neuron discharge timings, decoded from reinnervated residual muscles, into the forces simultaneously produced by 12 musculotendon units acting on a mechanical model of the amputee's missing limb (Fig. 7). The forces 
of the muscle-tendon units were concurrently projected to three degrees of freedom of the missing limb (elbow flexion/extension, forearm pronation/supination, wrist flexion/extension). The similarity between joint moments in the intact (as reference) and missing limb was quantified using both $R^{2}$ and the root mean squared difference (RMSD). Fig. 7 shows a representative example, which involved elbow flexion/extension $\left(R^{2}=0.82\right.$, $\mathrm{RMSD}=0.91 \mathrm{Nm})$, forearm rotation $\left(\mathrm{R}^{2}=0.77, \mathrm{RMSD}=0.04 \mathrm{Nm}\right)$, and wrist flexion/extension $\left(R^{2}=0.60, R M S D=0.02 \mathrm{Nm}\right)$, simultaneously. When decoding mechanical function for two degrees of freedom simultaneously - wrist rotation and flexion/extension - the performance corresponded to $\mathrm{R}^{2}=0.72, \mathrm{RMSD}=0.04 \mathrm{Nm}$, and $\mathrm{R}^{2}=0.73, \mathrm{RMSD}=0.05 \mathrm{Nm}$, respectively. When only a single degree of freedom was estimated (elbow flexion/extension), the performance corresponded to $\mathrm{R}^{2}=0.79, \mathrm{RMSD}=1.24 \mathrm{Nm}$.

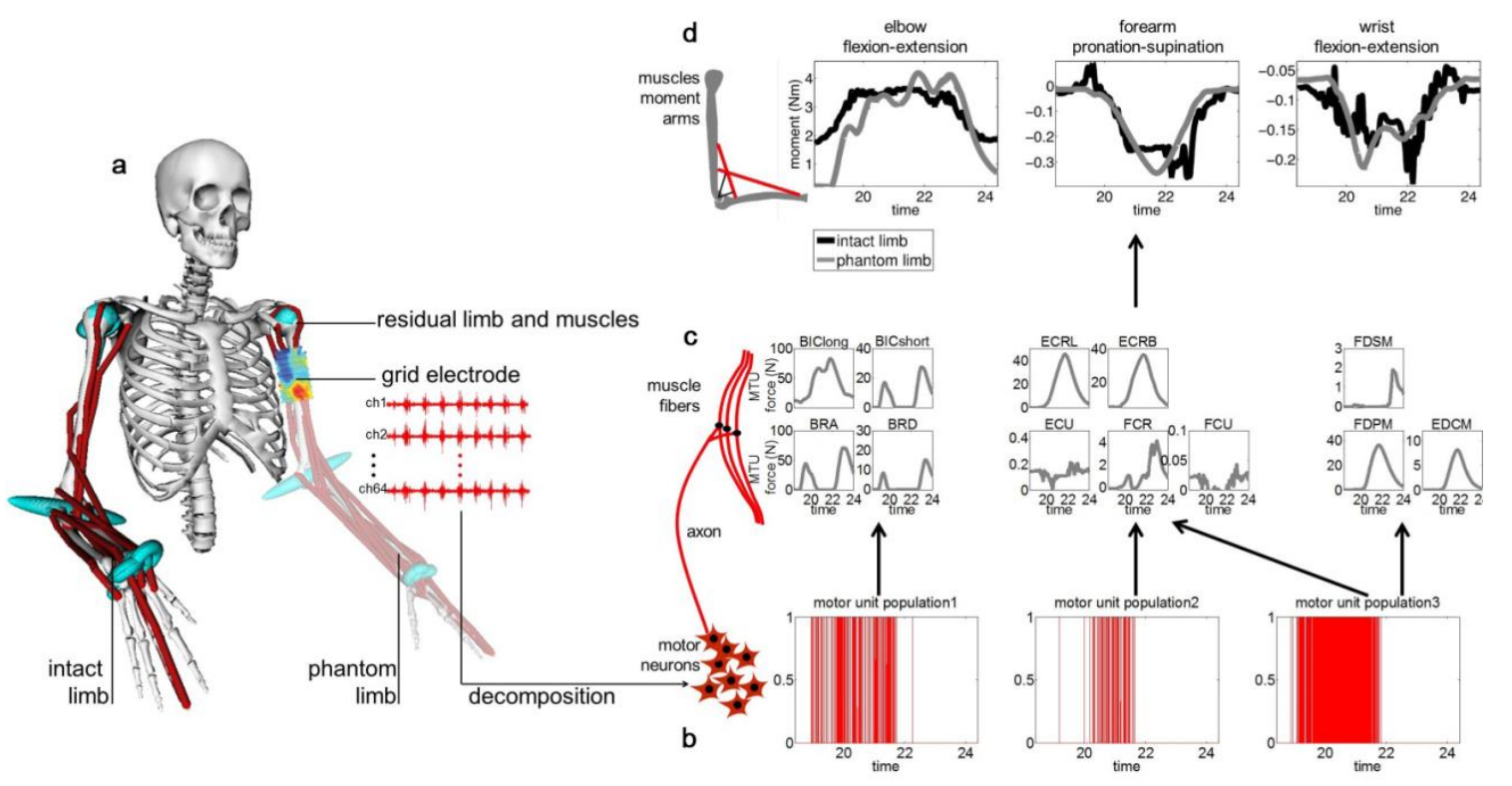

Figure 7 - (a) Subject-specific musculoskeletal geometry model built for patient T6. The model incorporates wire-like muscle-tendon units and wrapping surfaces to bones on both the intact and missing limb. One out of the three electrode grids used in the experiments is shown. (b) Decomposed motor neuron discharge timings are pooled into populations that represent the neural drive to 12 muscle-tendon units in the individual's missing limb. (c) The 
forces of the muscle-tendon units of the missing limb are estimated with the musculoskeletal model driven by motor neuron discharges. Muscles of the model are: biceps brachii long head (BIClong) and short head (BICshort), brachialis (BRA), brachioradialis (BRD), extensor carpi radialis longus (ECRL), extensor carpi radialis brevis (ECRB), extensor carpi ulnaris (ECU), flexor carpi radialis (FCR), flexor carpi ulnaris (FCU), flexor digitorum superficialis on middle finger (FDSM), flexor digitorum profundus on middle finger (FDPM) and extensor digitorum communis on middle finger (EDCM) (d) The muscle-tendon unit forces are simultaneously projected, via moment arms, to 3 degrees of freedom. Joint moments predicted in the missing limb are depicted together with those experimentally measured from the intact limb during a bi-lateral mirror task involving simultaneous elbow flexion, forearm pronation and wrist flexion. These results indicate the reconstruction of a missing limb biomechanics from the neural activity of the motor neuron pools which previously innervated the missing limb by using accessary muscles as an interface with these motor neurons.

These results illustrate the possibility, in principle, of estimating the biomechanics of a missing limb by decoding the neural drive to the muscle units and therefore the ultimate neural code representing the motor tasks. With this approach, predicted limb biomechanics can be translated into mechatronic functions of external devices that can be as complex as an intact biological limb.

\section{Discussion}

We have presented a new neural interface based on the decoding of the discharge timings of spinal motor neurons that provides the neural drive to muscles of missing limbs in amputees, to generate intuitive commands for potential prosthesis control. The interface is realized by the combination of TMR, that connects the axons of the target motor neurons to available muscle fibers, and the decoupling, by deconvolution, of the electrical activity of the muscle fibers innervated by each axon from multi-channel EMG recordings. We have shown that this interface enables the generation of meaningful and accurate control signals for potential prosthetic control, both in the context of pattern recognition with a large number of classes and within the paradigm of simultaneous and proportional control of multiple degrees of freedom. 
The proposed interface allowed detecting a large number of motor neurons (on average $>10$ ) for each reinnervation site. The series of discharges of these motor neurons could be accurately identified, so that the neural information sent from the pools of motor neurons previously innervating the missing muscles in the patients could be assessed. At a theoretical level, the decoded motor neuron behavior from EMG recordings may be considered a new EMG feature for use in myocontrol, however we consider it as conceptually different from any previous attempt for myocontrol. The main breakthrough is the change of analysis scale from macroscopic (EMG) to microscopic (timing of motor neuron discharges). Because the EMG is a signal generated by the spatio-temporal convolution of thousands of motor neuron discharges, any set of mathematically derived EMG features will reflect the temporal and spatial resolution of the EMG as a random process. In contrast, motor neuron discharge timings have a precise physiological meaning and represent the way in which movement is naturally coded at the spinal level. It is the same set of features that direct intrafascicular nerve interfacing attempts to extract ${ }^{5}$. Therefore, our results are on par with the identification of discharges directly from axonal interfacing with implanted intrafascicular electrodes. Nonetheless, with respect to implanted nerve recodings, the proposed system identifies a greater number of motor neurons and extracts the complete series of discharges of these motor neurons (see for comparison, e.g. Reference ${ }^{27}$ where the error rate is approximately $30 \%$ with at most 5-6 decoded efferent fibers from nerve interfacing). In this view, the proposed approach constitutes the ultimate exploitation of TMR as a means for neural interfacing using muscles as biological amplifiers. The results presented show that direct access to the neural code sent by peripheral nerves to muscles is possible and practical following TMR. Moreover, this study also shows that motor neuron spike trains can be effectively used as a relevant source of information for 
synthesizing complex prosthetic commands. In comparison, direct neural recordings have been usually applied to map commands using global features of the interference nerve signals without spike sorting (e.g., ${ }^{28}$ ).

The accuracy of the proposed interface has been proven with various control approaches and experimental designs on patients with amputations at different levels. First, we applied methods for signal classification, which are dominant in the scientific literature on myocontrol ${ }^{29}$, to the decoded series of discharge timings. The classification results indicated an almost perfect discrimination (on average $>97 \%$ ) of up to 11 classes. The results obtained from global EMG analysis in this study are partly in agreement with those reported in previous studies (e.g., ${ }^{15,19,30}$ ). However, given the relatively small number of available TMR patients in the current and previous studies and differences in the experimental and analysis conditions, comparisons between studies are difficult. For example, using classic EMG features, a classification accuracy of $98 \%$ and $93 \%$ was previously reported for two patients and 8 classes $^{30}$, which is a similar result as obtained in the present study for patients T1 and T2 for 7 and 9 classes, respectively. In our experiment, however, the performance with classic EMG features degraded for patient T3 when classifying 11 classes and was lower than that previously reported in four patients for 16 classes $^{19}$. However, three of the four patients in this previous study were transhumeral amputees with physiological innervation of the lateral heads of biceps and triceps and the window duration used for the analysis (256 ms) was longer than in our study $(100 \mathrm{~ms})$. In the experimental conditions of our study and with the choices we have made for the processing parameters, the proposed approach based on decoding motor neurons led to greater accuracy than the classic pattern recognition approach. Given the relatively small number of patients in this and previous studies, however, this result cannot be generalized. 
Furthermore, we tested the possibility for the patients to proportionally control one degree of freedom through decoded motor neuron activity (direct control). The decoded neural information allowed a finer separation of intensity levels than surface EMG amplitude. Surface EMG has indeed an associated estimation variance that is limited by the bandwidth of the signal ${ }^{31}$ while the extracted neural information can in principle exactly predict force because force is generated as a direct linear transformation of the decoded neural activity ${ }^{32}$. Accordingly, the variability in force control shown in Fig. 5 was lower when using the motor neuron discharge timings than with EMG amplitude in almost all conditions and patients tested. These results indicate the possibility of superior precision in proportional control by using the discharge patterns of motor neurons with respect to EMG amplitude.

The final experiment was related to the simultaneous and proportional control of multiple degrees of freedom, shown for two approaches. The signal-based approach identifies the control signals without labeling of the kinematics during training whereas the model-based approach is based on training and relies on the subject's anatomy. Both methods provided an estimate of the mechanics of multiple degrees of freedom. The TMR procedure allows the full reconstruction of the neural signals sent to the muscles of the missing limb, despite the absence of these muscles, so that the biomechanics of the missing limb can be estimated, in principle, accurately. This experiment was performed on only one of the patients, due to limited availability of the other patients. However, the patient who participated in this test did not show EMG signals of better quality than the other patients and, importantly, the decomposition was of the same quality in this and other patients. Moreover, the patients who participated in the second experiment, including the one who took part to the third experiment, had very similar performance in their motor neuron 
proportional control (Fig. 5). Therefore, the patient selected for the third experiment on simultaneous and proportional control was similar to the others for the type and quality of neural information extracted and for the control he could achieve using this neural information in a previous experiment. There is no reason to believe that the results of the third experiment are specific to the patient selected.

The superior performance of the use of motor neuron discharge timings with respect to global EMG in some of the conditions analyzed has several biophysical reasons. The global EMG features vary for changes in the waveforms of muscle fiber action potentials. This factor of variability is removed by separating the neural information from the muscle fiber action potentials. Moreover, the decoding method does not use clustering based on the shape of the action potentials but rather identifies the discharge timings by sparseness constraints (see Methods). Therefore, changes in the action potential shapes do not influence the decomposition. The separation of action potentials from the neural drive also eliminates the effect of EMG amplitude cancellation ${ }^{31}$, which poses an intrinsic limit to the accuracy of EMG amplitude estimates. This partly explains, for example, the observations of the second experiment. In the specific clinical case of TMR patients, there are additional reasons for the effectiveness of the proposed approach with respect to global EMG analysis. The discharge patterns of reinnervated motor units are likely easier to discriminate than for physiological innervaton due to a reduced complexity of the interference signal. Moreover, the reinnervation causes a loss of the natural relation between muscle unit size and recruitment order so that the amplitude of the EMG signals is likely a poorer indicator of muscle activation in these patients than in physiological conditions, as suggested in ${ }^{23}$. Finally, the territories of the muscle units of different motor neuron pools tends to overlap during 
the reinnervation process and their activities are therefore difficult to differentiate from the global EMG analysis.

The proposed interface can presumably be applied to any nerve that can be redirected to accessory muscles. Even in the absence of target muscle tissue, this may be obtained either with transplants of small muscle portions ${ }^{33}$ or, in the future, by growing muscle tissue around the terminal portion of the nerve ${ }^{34}$. Therefore, the proposed system should be seen as a general neural interface that accurately decodes the efferent activity of nerves without inserting electrodes into nerves. Moreover, although the current system was tested with non-invasive EMG recordings, the same concepts and algorithms can be directly translated to implanted multi-site muscle electrodes, such as the intramuscular arrays we have recently proposed ${ }^{35}$ or multi-channel extensions of epimysial devices already tested in patients ${ }^{36}$. These muscle implants could be surgically realized at the same time as the TMR procedure.

Although obtained under several experimental conditions and on patients with different characteristics, the presented results are currently limited to an offline analysis. The training and use of the proposed interface needs to be performed online and should explore the perception action cycle of the user in a closed-loop scenario to fine tune motor control to achieve the desired movements. While these future steps are needed for a full appreciation of the potential of the approach, the current work provides proof of the feasibility of decoding motor neuron behavior in amputees and of using motor neuron behavior for man-machine interfacing.

The online implementation of the proposed concepts for the control of prostheses in daily-life activities requires the solution of practical challenges. The first of these challenges 
is the requirement for an online EMG deconvolution that is computationally complex. Nonetheless, this operation is now feasible due to speed of current microprocessors ${ }^{37}$. The performance with the online user's control may differ from the offline results ${ }^{38}$. However, because motor neurons acting on a degree of freedom receive predominantly common input $^{39}$, the identification of different sets of motor neurons in different sessions would not influence the mapping and control. Despite these promising features, the performance of the system over different conditions without re-training during online use has not been tested and constitutes a challenge for the clinical translation. Morever, the clinical efficacy of any of the control schemes proposed will depend on the design of the mechatronics of a given prosthetic limb. The current study focuses on an extensive offline experimental validation on patients that provides a proof-of-concept for an innovation in myocontrol whose clinical translation needs further efforts.

In conclusion, we have proven the possibility of decoding the behavior of virtually all the pools of motor neurons that physiologically innervated the muscles responsible for the movement of a missing limb and that are reinnervated to other muscle tissues by TMR. This decoding was demonstrated in six patients with different amputation levels and TMR procedures, and was shown to provide information that can be used for intuitive and effective commands over multiple degrees of freedom. The approach is the ultimate exploitation of the TMR concept that allows spinal interfacing by using muscles as natural amplifiers of nerve activity. A full clinical translation of this new concept requires online implementation of the proposed algorithms and testing the long-term adaptation with the user in the loop.

\section{Methods}




\section{Patients}

Measurements were performed on six TMR patients, whose characteristics relevant for this study are reported in Table 1. The patients were referred to O.C.A. for prosthetic fitting and underwent TMR surgery. The characteristics of the patients reported in Table 1 refer to the time when each patient was included in the experimental measurements. The TMR procedures for all patients were performed at the Medical University of Vienna, Austria. In patients $\mathrm{T} 1, \mathrm{~T} 2, \mathrm{~T} 3$, all major nerves of the brachial plexus were redirected into muscles of the chest region (Table 1). Patients T4, T5 and T6 are transhumeral amputees for whom the TMR procedure resulted in the medianus, ulnaris, and radialis nerves being reinnervated into the brachialis, caput breve bicipitis, and caput laterale tricipitis muscles, respectively.

The experimental protocols as well as the informed consent forms for the experiments were approved by the ethics committee "Ethikkommission der Medizinischen Universität Wien" (approval numbers 1279/2014, for patients T1-T3, and 1234/2015, for patients T4-T6).

\section{Experimental set-up}

Three experiments were performed with the aim of showing different control strategies for active prostheses, all based on the decoded activity of the motor neurons reinnervating muscles above the amputation. The patient selection for each experiment was not randomized since the measurements were performed over the course of two years and designed with the purpose of presenting progressively more advanced features of the proposed interface. The six patients participated in the different experiments depending on their availability at the time when the methods applied in the three experiments were 
developed. Therefore, the experimental work represents a case series of several experimental sessions on a total of six patients with the purpose of presenting the concept of spinal interfacing via TMR and motor neuron activity decoding. Fig. 1 shows the general concept underlying the methods applied in all experiments. Grids of 64 electrodes for surface EMG recordings were located over each reinnervation point. The multi-channel EMG signals were processed to extract the series of discharges of the innervating motor neurons ${ }^{24,40}$. The identified spike trains were then used to define control commands. The mapping between neural information and motion of degrees of freedom varied in the three experiments, as described in the following, with the aim of showing different control solutions.

\section{EMG recording and processing}

In all of the experiments, surface EMG signals were recorded with high-density and flexible electrode grids. The placement of the grids differed across individuals and corresponded to the locations of reinnervation following surgery. The EMG electrode grids incorporate copper tracks on a kapton support and each grid comprises 64 sensors $(8 \times 8$ electrodes, 1-mm diameter; Spes Medica, Italy) with an inter-electrode distance of $10 \mathrm{~mm}$ in both directions. The grids were applied on the skin surface by a 1-mm thick double adhesive foam with holes in correspondence to the electrode locations. The skin-electrode contact was facilitated by the use of conductive paste. The multi-channel signals were amplified by a multi-channel amplifier (EMGUSB2, OTBioelettronica, Italy, cutoff frequencies 3-900 Hz), sampled at $2048 \mathrm{~Hz}$, and A/D converted with 12 bit precision. Although a surface recording system was chosen for this study, the same processing methods and experimental tests can be also applied with implanted muscle electrodes ${ }^{40}$. The only fundamental characteristics of 
the EMG recording for the proposed approach is the availability of several (>30) channels per reinnervated site, as it can be achieved with invasive technology by, e.g., our recently developed thin-film electrodes ${ }^{35}$.

The EMG signals were decomposed by a blind source separation algorithm ${ }^{40}$. The decomposition provided the discharges of activation of the innervating motor neurons. The decomposition algorithm is described in detail in ${ }^{40}$ but we also provide here a brief description of its basic working principles.

Multi-channel EMG signals are observations that contain the convolutive mixtures of motor neuron spike trains. For each observation (EMG channel), unknown finite impulse response filters (motor unit action potentials) act on the sources (series of motor neuron discharge timings). This convolutive mixture of sources can be converted into a linear instantaneous mixture by extending the sources to include the $n$ original sources as well as their delayed versions, with delays from 1 to the filter length $L^{40}$. The $m$ original observations (EMG channels) are also extended, by a factor $R$, in order to maintain a greater number of observations than sources.

$$
\text { Indicating with } s(k)=\left[s_{1}(k), s_{2}(k), \ldots, s_{n}(k)\right]^{T}, x(k)=\left[x_{1}(k), x_{2}(k), \ldots, x_{m}(k)\right]^{T}
$$

and $n(k)=\left[n_{1}(k), n_{2}(k), \ldots, n_{m}(k)\right]^{T}$ the original sources, observations, and noise, respectively, the extended model is as follows:

$$
x(k)=[H s(k)+n(k)] \quad k=0, \ldots, D_{R}
$$

where the extended observations, sources, and noise are:

$$
\begin{gathered}
\widetilde{x_{l}}(k)=\left[x_{i}(k), x_{i}(k-1), \ldots, x_{i}(k-R)\right] i=1, \ldots, m \\
\widetilde{s_{J}}(k)=\left[s_{j}(k), s_{j}(k-1), \ldots, s_{j}(k-L-R)\right] j=1, \ldots, n
\end{gathered}
$$




$$
\widetilde{n_{l}}(k)=\left[n_{i}(k), n_{i}(k-1), \ldots, n_{i}(k-R)\right] \quad i=1, \ldots, m
$$

and the equivalent scalar mixing matrix representing the filters is:

$$
\underline{\underline{H}}=\left[\begin{array}{ccc}
\widetilde{h_{11}} & \cdots & \widetilde{h_{1 n}} \\
\vdots & \ddots & \vdots \\
\widetilde{h_{m 1}} & \cdots & \widetilde{h_{m n}}
\end{array}\right]
$$

with:

$$
h_{i j}=\left[\begin{array}{cccccc}
h_{i j}[0] & \cdots & h_{i j}[L-1] & 0 & \cdots & 0 \\
0 & \ddots & \ddots & \ddots & \ddots & \vdots \\
\vdots & \ddots & \ddots & \ddots & \ddots & 0 \\
0 & \cdots & 0 & h_{i j}[0] & \cdots & h_{i j}[L-1]
\end{array}\right]
$$

Where $D_{r}$ is the duration of the recordings and $h_{i j}$ the action potential of the $j$-th motor unit recorded at the channel $i$. The noise in model (1) represents electronic noise as well as the activity of motor units represented at the skin surface by low-energy action potentials that are not separated.

The linear instantaneous model of Eq. (1) is inverted to recover the matrix of the extended sources. In this study, the inversion is performed by spatial whitening followed by the fixed point optimization procedure with a cost function that maximizes the sparseness of the estimated sources ${ }^{40,41}$. The method extracts sources (series of motor neuron discharge timings) associated to individual motor neurons, as proven by the unique represention of the associated multi-channel surface action potentials ${ }^{42}$. The estimated sources are trains of delta functions centered at the instant of motor neuron activation, with an amplitude that may vary due to the estimation process. In order to extract the discharge timings information only, a local peak detector was applied to the estimated sources, by comparing each candidate peak with surrounding peaks, and considering a refractory period of $10 \mathrm{~ms}$. It is relevant to note that the decomposition method extracts the original sources as well as 
their delayed replicas, as defined by the extended source model of Eq. (3). For all analyses performed in this study, the delayed replicas were excluded and only the estimated original sources were further processed for the mapping procedures. The only exception is the signal-based mapping for simultaneous and proportional control of multiple degrees of freedom based on principal component analysis (PCA) (Experiment 3) for which we used as input the full extended estimated sources as in Eq. (3). For this reason, the number of time series of discharge timings used as input with that approach is much greater than for the others ( $L$ times greater).

Applying the above decomposition procedure, Fig. 1 shows an example of sets of discharge times of populations of motor neurons during tasks of wrist flexion and extension by a patient following TMR. These discharge timings are not abstract mathematical features, as in classic myocontrol, but instead correspond to the natural neural code sent from the spinal cord to the muscles.

After EMG decomposition, the neural drive to muscles was estimated by pooling the discharge timings of groups of identified motor neurons ${ }^{39,43}$. The selection of these groups depended on the analysis and is detailed for each experiment in the following. For each group of motor neurons, the corresponding neural drive was the ensemble of discharge timings of the motor neurons. The signal of delta functions centered at each discharge of activation of each motor neuron in a defined group of neurons represents all timings of discharge of the motor neurons in the group. It is simply obtained by summing the series of discharge timings of the motor neurons in the group and corresponds physiologically to the neural drive sent to the reinnervated muscles by the defined motor neuron group. The estimated neural drives to the reinnervated muscles were then used to map control signals 
into multiple degrees of freedom in three experiments. The common procedures used in all experiments for EMG processing to extract the neural activation signals are schematically shown in Fig. 1.

\section{Experiment 1: Classification}

Patients T1-T3 (Table 1) participated in experiment 1 . The patients were asked to attempt the following tasks of their missing limb: hand opening, hand closing, wrist extension, wrist flexion, thumb adduction, thumb abduction, pronation, supination, elbow extension, elbow flexion. Due to different reinnervation profiles and levels of training, not all subjects were able to perform all these tasks. The order of the attempts was randomized. For patient T1 each attempt lasted $10 \mathrm{~s}$, for patients T2 and T3 the trials lasted $5 \mathrm{~s}$, with $5 \mathrm{~s}$ of rest in between trials. Patients T1 and T2 performed each trial twice, while patient T3 repeated each trial three times. These differences in experimental choices for the different patients were due to the conditions and capabilities of the patients.

The neural drives to muscle regions were estimated, as described above, by pooling the discharge timings of groups of motor neurons. The groups were defined based on the location of the muscle units in the areas covered by the recording electrode grids. Each grid was divided into four regions and each motor neuron was associated to the muscle unit in the region of the grid where the corresponding motor unit action potential had the greatest amplitude. In this way, we defined the neural drives to reinnervated muscle sites by partitioning each grid into four parts and associating to them the ensembles of discharges of the innervating motor neurons.

Classification into the discrete classes was performed using a support vector machine classifier with linear kernel, with 5-fold cross-validation. The input to the classifier was the 
number of discharges in each 100 -ms interval for each muscle region, with a 10 -ms overlap between consecutive intervals. For comparison with surface EMG classification, the EMG RMS of all channels as well as the TD features were computed in the same intervals used for spike classification and classified with the same classifier. For global EMG features, the feature space was reduced in dimensionality using Principal Component Analysis (PCA), retaining $95 \%$ of the signal power.

\section{Experiment 2: Direct control}

The aim of experiment 2 was to establish if it was possible to extract a proportional command from motor unit discharges (direct control). The experiment was performed on patients $\mathrm{T} 4, \mathrm{~T} 5$ and $\mathrm{T} 6$, who are all transhumeral amputees (Table 1). The patients were seated comfortably facing a computer screen. One surface EMG electrode grid was mounted over the reinnervated short head of the biceps. Initially, all participants were asked to perform a maximal voluntary contraction by attempting a hand open gesture of their missing limb. The maximum EMG envelope during this task was taken as reference for providing feedback in percent of the maximum intensity. Each subject was then prompted to increase and decrease the intensity of muscle activity from the relaxed state to the maximal intensity, over $20 \mathrm{~s}$. This contraction duration determined a slow-varying force contraction. This choice was made in order to assess accurate control, which requires slow variations, and in the full activation range of the muscle, to prove that the analysis methods proposed are not influenced by the strength of activation. The contractions performed by the patients represent accurate force control, as it can be seen in fine object manipulation. Conversely, Experiment 3 focused on faster contractions, as typical of reaching tasks. 
The intensity of muscle activation was estimated using the EMG envelope, as a classic reference approach, and the neural drive, as direct neural information. These approaches were compared varying the processing interval from $50 \mathrm{~ms}$ to $500 \mathrm{~ms}$, with an interval overlap of $50 \%$. Moreover, a post processing was applied by averaging over three consecutive past intervals. For each condition, the standard deviation of the intensity estimate, after linear de-trending, was computed to determine the accuracy in the control.

\section{Experiment 3: Control of multiple degrees of freedom}

Among the tested patients, patient T6 (Table 1) volunteed for a further experimental session that involved attempted mirror movements of the missing and the contralateral arm with the purpose of testing the ability of the proposed approach to map the neural drive into multiple degrees of freedom activated concurrently during natural movements. For this purpose, motion capture data and EMG signals were recorded concurrently. Upper limb kinematics were recorded ( $256 \mathrm{~Hz}$ sampling frequency) using a seven-camera system (Qualisys, Göteborg, Sweden) and a set of 18 retro-reflective markers placed on the patient's intact left upper extremity, residual right upper extremity, trunk, and pelvis. High-density EMG was recorded using electrode grids located in correspondence of the residual upper arm frontal, lateral, and dorsal compartments to cover the targeted reinnervations. Data were recorded during one static anatomical pose and 13 dynamic trials involving mirrored bi-lateral motions that simultaneously articulated elbow flexion, forearm pronation, and wrist flexion, both in the intact and missing limb. These three degrees of freedom were chosen since in transhumeral amputees the precise proportional and simultaneous control over elbow and wrist is the biggest challenge in prosthetic fitting. Precise proportional and simultaneous control over these three degrees of freedom allows natural object reaching for 
transhumeral amputees, which is not possible with current systems. The hand openingclosing task was not included in this experiment since this can be achieved without simultaneous control, by an hybrid scheme as proposed in ${ }^{7}$, whereas the focus of this experiment was on concurrent and proportional activation of degrees of freedom. The estimate of the kinematics from neural information was performed with both a signal-based and a model-based estimation approach.

Signal-based estimation. An approach for extracting control signals using unsupervised subspace mapping was developed, which receives as input the full extended sources [Eq. (3)] and transforms them in direct control signals. The central idea of the method is to preserve the metric of the high-dimensional space where the multidimensional time series of motor neuron dischages exists and to project the data to a subspace of dimension specified by the degrees of freedom of the prosthetic hand. The simplest of the metric projections uses the Mahalanobis distance ${ }^{36}$, which is defined by the covariance of discharge time series. Principal component analysis (PCA) was used to explore the spatiotemporal correlation between the time series in high-dimensional space and to project the data to an orthogonal lower dimensional space. Let $\mathbf{X}_{n \times D}$, be the input data matrix representing the $n$ discharge time series over $D$ channels. Then PCA performs a singular value decomposition (SVD) of $\mathbf{X}=\mathbf{U} \mathbf{\Sigma} \mathbf{V}^{\mathrm{T}}$, where $\mathbf{U}$ and $\mathbf{V}$ are $n \times n$ and $D \times D$ unitary matrices, respectively, and $\boldsymbol{\Sigma}$ is a $n \times D$ rectangular diagonal matrix with diagonal values $\sigma_{i}$ known as the singular values of $\mathbf{X}$. The columns of $\mathbf{V}$ are the eigenvectors of the covariance matrix and they serve as the principal directions or axes of the PCA sub-space. The principal components $\mathrm{U} \Sigma$ are projections of $\mathbf{X}$ on sub-space axes. PCA achieves a projection that maps most of the relevant information (variance) to a $d$-dimensional manifold, where $d(d \ll D)$ is any suspace. It can be shown that this mapping is formed by the first $d$ eigenvectors, i.e., 
columns of $\mathbf{V}$, noted as $\mathbf{V}_{d}$. In our case, $d$ is specified by the number of degrees of freedom to be controlled, and the goal is to associate each principal component to a degree of freedom. Therefore, the problem reduces to appropriately assigning the outputs of the PCA projected data to the corresponding degree of freedom. However, the two orthogonal coordinate systems (that of PCA and that of the degrees of freedom) of the same dimension are not necessarily the same because the PCA eigenvectors are solely defined by the data, hence an orthogonal rotation $\mathbf{R}_{d \times d}$ is needed to align the principal directions with the external basis of degrees of freedom. Moreover, the external basis coordinates are the canonical sparse basis of $R^{n}$ (each component only moves one degree of freedom of the prosthesis), while the PCA eigenvectors are not sparse and are ordered by projected variance. Therefore, we sought to find the rotation matrix in the principal component subspace that is the most sparsified to mimic the canonical basis of $R^{n}$. Here, we applied the VARIMAX orthogonal rotation ${ }^{37,38} \mathbf{R}_{\text {VARIMAX: }}$

$$
\mathbf{R}_{\text {VARIMAX }}=\arg \max _{R}\left(\frac{1}{d} \sum_{j=1}^{n} \sum_{i=1}^{d}(\mathbf{L R})_{i j}^{4}-\sum_{j=1}^{n}\left(\frac{1}{d} \sum_{i=1}^{d}(\mathbf{L R})_{i j}^{2}\right)^{2}\right)
$$

where $\mathbf{L}_{n \times d}=\boldsymbol{\Sigma}_{n \times d} \mathbf{V}_{d \times d} /(\sqrt{n-1})$ are referred to as the loadings ${ }^{37}$. Once the PCA and VARIMAX rotation are obtained in a training set (without any kinematic labeling), the control of the prosthesis test is achieved by inputing the motor neuron discharge time series in real time to the PCA subspace and VARIMAX projections, which can be combined to decrease the computational complexity.

Model-based estimation. The open-source software OpenSim ${ }^{44}$ was used to scale a generic upper extremity model of the musculoskeletal geometry ${ }^{45}$ to match the patient's anthropometry. The musculoskeletal geometry model had seven upper extremity degrees of 
freedom and incorporated a total of 14 muscle-tendon units, spanning the shoulder, elbow, wrist and hand joints (Fig. 7). During the scaling process, virtual markers were placed on the generic musculoskeletal geometry model based on the position of the experimental markers from the static pose. The model anthropomorphic properties as well as the muscle-tendon unit insertion, origin and their bone wrapping points were linearly scaled on the basis of the relative distances between experimental and corresponding virtual markers ${ }^{44}$. Inverse kinematics was solved for three-dimensional joint angles that minimized the least-squared error between experimental and virtual marker locations during dynamic trials ${ }^{46}$. The generated kinematics were then used to obtain dynamically consistent joint moments via residual reduction analysis, i.e. joint moments reconstructing experimental joint angles when driving forward dynamics arm simulations ${ }^{47}$. We refer to these as "experimental joint moments".

The estimates of joint moments were based on neural data-driven musculoskeletal modeling. The motor neuron discharges were converted into continuous neural activations using a twitch model based on a time-history dependent recursive filter and a non-linear transfer function ${ }^{48}$. Experimental joint angles were used as input to a multidimensional cubic B-splines set that synthetized the OpenSim subject-specific geometry of muscle-tendon units and computed their resulting length and moment $\operatorname{arms}^{49,50}$. Neural activations and muscletendon unit length were used to control a Hill-type muscle model and estimate instantaneous length, contraction velocity, and force in the muscle fibers, and strain and force in the series-elastic tendon within each muscle-tendon unit ${ }^{49}$. The computed forces were projected onto all upper extremity degrees of freedom simultaneously via the moment arms. 
The neural-driven model was calibrated to map neural activations to individual muscle-tendon units. After the calibration, the model was used to convert neural inputs into the resulting joint moments produced in the missing limb elbow, forearm and wrist.

\section{Data availability}

The data and the codes used for the results presented in this study are available upon request. 


\section{References}

1. Farina, D. \& Aszmann, O. Bionic limbs: clinical reality and academic promises. Sci. Transl. Med. 6, 257ps12 (2014).

2. Kuiken, $\mathrm{T}$. a et al. Targeted reinnervation for enhanced prosthetic arm function in a woman with a proximal amputation: a case study. Lancet 369, 371-80 (2007).

3. Tan, D. W. et al. A neural interface provides long-term stable natural touch perception. Sci. Transl. Med. 6, 257ra138-257ra138 (2014).

4. Lebedev, M. A. \& Nicolelis, M. A. L. Brain-machine interfaces: past, present and future. Trends Neurosci. 29, 536-546 (2006).

5. Dhillon, G. S., Lawrence, S. M., Hutchinson, D. T. \& Horch, K. W. Residual function in peripheral nerve stumps of amputees: implications for neural control of artificial limbs. $J$. Hand Surg. Am. 29, 605-15-8 (2004).

6. Wodlinger, B. \& Durand, D. M. Peripheral nerve signal recording and processing for artificial limb control. 2010 Annu. Int. Conf. IEEE Eng. Med. Biol. Soc. EMBC'10 6206-6209 (2010). doi:10.1109/IEMBS.2010.5627735

7. Amsuess, S. et al. Context-Dependent Upper Limb Prosthesis Control for Natural and Robust Use. IEEE Trans. Neural Syst. Rehabil. Eng. 1-1 (2015). doi:10.1109/TNSRE.2015.2454240

8. Gilja, V. et al. A high-performance neural prosthesis enabled by control algorithm design. Nat. Neurosci. 15, 1752-7 (2012).

9. Donoghue, J. P. Connecting cortex to machines: recent advances in brain interfaces. Nat. Neurosci. 5 Suppl, 1085-8 (2002).

10. Hochberg, L. R. et al. Reach and grasp by people with tetraplegia using a neurally controlled robotic arm. Nature 485, 372-375 (2012).

11. Collinger, J. L. et al. High-performance neuroprosthetic control by an individual with tetraplegia. Lancet 381, 557-564 (2013).

12. Bouton, C. E. et al. Restoring cortical control of functional movement in a human with quadriplegia. Nature 533, 247-250 (2016).

13. Jiang, N., Dosen, S. \& Farina, D. Myoelectric control of artificial limbs: is there the need for a change of focus? IEEE Signal Process. Mag. 12-15 (2012).

14. Hoffer, J. \& Loeb, G. Implantable electrical and mechanical interfaces with nerve and muscle. Ann. Biomed. Eng. 8, (1980).

15. Kuiken, T. a et al. Targeted muscle reinnervation for real-time myoelectric control of multifunction artificial arms. JAMA 301, 619-628 (2009).

16. Gart, M. S., Souza, J. M. \& Dumanian, G. A. Targeted Muscle Reinnervation in the Upper Extremity Amputee: A Technical Roadmap. J. Hand Surg. Am. 40, 1877-1888 (2015).

17. Kuiken, $\mathrm{T}$. a et al. The use of targeted muscle reinnervation for improved myoelectric prosthesis control in a bilateral shoulder disarticulation amputee. Prosthet. Orthot. Int. 28, 245-253 (2004). 
18. Cheesborough, J. E., Dumanian, G. A., Smith, L. H. \& Kuiken, T. A. Targeted Muscle Reinnervation and Advanced Prosthetic Arms. Semin. Plast. Surg. 1, 62-72 (2015).

19. Zhou, P. et al. Decoding a new neural machine interface for control of artificial limbs. J. Neurophysiol. 98, 2974-82 (2007).

20. Tikhonov, A. N. On the stability of inverse problems. Dokl. Akad. Nauk SSSR 39, 195-198 (1943).

21. Birdwell, J. A., Hargrove, L. J., Weir, R. F. \& Kuiken, T. A. Extrinsic finger and thumb muscles command a virtual hand to allow individual finger and grasp control. IEEE Trans. Biomed. Eng. 62, 218-226 (2015).

22. Cipriani, C., Segil, J. L., Birdwell, J. A. \& Weir, R. F. Dexterous control of a prosthetic hand using fine-wire intramuscular electrodes in targeted extrinsic muscles. IEEE Trans. Neural Syst. Rehabil. Eng. 22, 828-836 (2014).

23. Li, Y., Smith, L. H., Hargrove, L. J., Weber, D. J. \& Loeb, G. E. Sparse optimal motor estimation (SOME) for extracting commands for prosthetic limbs. IEEE Trans. Neural Syst. Rehabil. Eng. 21, 104-111 (2013).

24. Farina, D. et al. Non-Invasive, Accurate Assessment of the Behavior of Representative Populations of Motor Units in Targeted Reinnervated Muscles. IEEE Trans. Neural Syst. Rehabil. Eng. 4320, (2014).

25. Kapelner, T., Jiang, N., Vujaklija, I., Aszmann, O. C. \& Farina, D. Classification of Motor Unit Activity Following Targeted Muscle Reinnervation. in IEEE engineering in medicine and biology magazine : the quarterly magazine of the Engineering in Medicine \& Biology Society 22-24 (2015).

26. Holobar, A., Glaser, V., Gallego, J. A., Dideriksen, J. L. \& Farina, D. Noninvasive analysis of motor unit behavior in pathological tremor. Proc. Annu. Int. Conf. IEEE Eng. Med. Biol. Soc. EMBS 7512-7515 (2011). doi:10.1109/IEMBS.2011.6091852

27. Djilas, M., Azevedo-Coste, C., Guiraud, D. \& Yoshida, K. Spike sorting of muscle spindle afferent nerve activity recorded with thin-film intrafascicular electrodes. Comput. Intell. Neurosci. 2010, (2010).

28. Jezernik, S., Grill, W. W. \& Sinkjaer, T. Neural network classification of nerve activity recorded in a mixed nerve. Neurol. Res. 23, 429-434 (2001).

29. Parker, P., Englehart, K. \& Hudgins, B. Myoelectric signal processing for control of powered limb prostheses. J. Electromyogr. Kinesiol. 16, 541-548 (2006).

30. Hargrove, L., Ping Zhou, Englehart, K. \& Kuiken, T. A. The Effect of ECG Interference on Pattern-Recognition-Based Myoelectric Control for Targeted Muscle Reinnervated Patients. IEEE Trans. Biomed. Eng. 56, 2197-2201 (2009).

31. Farina, D., Merletti, R. \& Enoka, R. M. The extraction of neural strategies from the surface EMG. J. Appl. Physiol. 96, 1486-1495 (2004).

32. Negro, F., Holobar, A. \& Farina, D. Fluctuations in isometric muscle force can be described by one linear projection of low-frequency components of motor unit discharge rates. J. Physiol. 587, 5925-5938 (2009). 
33. Aszmann, O. C. et al. Bionic reconstruction to restore hand function after brachial plexus injury : a case series of three patients. Lancet 6736, 1-7 (2015).

34. Ursu, D. C., Urbanchek, M. G., Nedic, A., Cederna, P. S. \& Gillespie, R. B. In vivo characterization of regenerative peripheral nerve interface function. J. Neural Eng. 13, 26012 (2016).

35. Muceli, S. et al. Accurate and representative decoding of the neural drive to muscles in humans with multi-channel intramuscular thin-film electrodes. J. Physiol. n/a-n/a (2015). doi:10.1113/JP270902

36. Ortiz-Catalan, M., Hakansson, B. \& Branemark, R. An osseointegrated human-machine gateway for long-term sensory feedback and motor control of artificial limbs. Sci. Transl. Med. 6, 257re6-257re6 (2014).

37. Glaser, V., Holobar, A. \& Zazula, D. Real-time motor unit identification from high-density surface EMG. IEEE Trans. Neural Syst. Rehabil. Eng. 21, 949-958 (2013).

38. Jiang, N., Vujaklija, I., Rehbaum, H., Graimann, B. \& Farina, D. Is accurate mapping of EMG signals on kinematics needed for precise online myoelectric control? IEEE Trans. Neural Syst. Rehabil. Eng. 22, 549-58 (2014).

39. Farina, D., Negro, F. \& Dideriksen, J. L. The effective neural drive to muscles is the common synaptic input to motor neurons. J. Physiol. 49, 1-37 (2014).

40. Negro, F., Muceli, S., Castronovo, A. M., Holobar, A. \& Farina, D. Multi-channel intramuscular and surface EMG decomposition by convolutive blind source separation. J. Neural Eng. 13, 26027 (2016).

41. Farina, D. \& Holobar, A. Characterization of Human Motor Units From Surface EMG Decomposition. Proc. IEEE 104, 353-373 (2016).

42. Farina, D., Negro, F., Gazzoni, M. \& Enoka, R. M. Detecting the Unique Representation of Motor-Unit Action Potentials in the Surface Electromyogram. J. Neurophysiol. 100, 1223-1233 (2008).

43. Negro, F. \& Farina, D. Factors Influencing the Estimates of Correlation between Motor Unit Activities in Humans. PLoS One 7, (2012).

44. Delp, S. L. et al. OpenSim: Open-source software to create and analyze dynamic simulations of movement. IEEE Trans. Biomed. Eng. 54, 1940-1950 (2007).

45. Saul, K. R. et al. Benchmarking of dynamic simulation predictions in two software platforms using an upper limb musculoskeletal model. Comput. Methods Biomech. Biomed. Engin. 1-14 (2014). doi:10.1080/10255842.2014.916698

46. Lu, T. W. \& O'Connor, J. J. Bone position estimation from skin marker co-ordinates using global optimisation with joint constraints. J. Biomech. 32, 129-134 (1999).

47. Thelen, D. G. \& Anderson, F. C. Using computed muscle control to generate forward dynamic simulations of human walking from experimental data. J. Biomech. 39, 1107-1115 (2006).

48. Manal, K. \& Buchanan, T. S. A one-parameter neural activation to muscle activation model: Estimating isometric joint moments from electromyograms. J. Biomech. 36, 1197-1202 (2003). 
49. Sartori, M., Reggiani, M., Farina, D. \& Lloyd, D. G. EMG-driven forward-dynamic estimation of muscle force and joint moment about multiple degrees of freedom in the human lower extremity. PLoS One 7, e52618 (2012).

50. Sartori, M., Farina, D. \& Lloyd, D. G. Hybrid neuromusculoskeletal modeling to best track joint moments using a balance between muscle excitations derived from electromyograms and optimization. J. Biomech. 47, 3613-3621 (2014).

Acknowledgements: This work was supported by the European Research Council Advanced Grant DEMOVE (contract \#267888) (to D.F.), by the Christian Doppler Research Foundation of the Austrian Federal Ministry of Science, Research and Economy (to O.C.A.), by the European Union's Horizon 2020 research and innovation programme under the Marie Skłodowska-Curie grant agreement No 702491 (NeuralCon) (to F.N.), and DARPA HAPTIX (N66001-15-1-4054) (to J.P.). The authors are grateful to Drs. Meike Schweisfurth and Hubertus Rehbaum for support in the experimental measurements, to Dr. Margherita Castronovo for support in the data analysis, and to Drs. Christian Hofer and Stefan Salminger for clinical support.

Author contributions: D.F. and O.C.A. conceived the study. I.V., T.K., M.S. and K.B. performed the acquisition. I.V., T.K., M.S., F.N., N.J., A.A. and J.P. conducted the analysis. D.F., I.V., T.K., M.S., F.N., N.J., K.B., J.P. and O.C.A. interpreted the data. D.F., I.V. and O.C.A. wrote and edited the manuscript.

\section{Additional information:}

*Address of Correspondence:

Prof. Dr. Dario Farina

Department of Bioengineering

Imperial College London

SW7 2AZ London, UK

Tel: +44 (0)20 75941387

Email: d.farina@imperial.ac.uk

Competing interests: The authors declare no financial competing interests. 